AC 2007-2976: A PRACTICAL APPROACH TO INTEGRATE TEACHING VOICE-OVER-IP TECHNOLOGY IN THE CLASSROOM

Farid Farahmand, farahmandfar@ccsu.edu 


\section{A Practical Approach to Integrate Teaching Voice-over-IP Technology in the Classroom}

\section{Introduction}

Voice-Over-IP (VoIP), also called IP telephony, Internet Telephony, and Digital Phone, is simply transporting voice traffic using the Internet Protocol (IP). The Internet Protocol has become the de facto standard for data transactions and its ubiquitous presence has made it a suitable choice for transporting voice and video. VoIP technology offers many attractive advantages over the legacy telephony, including lower equipment cost, lower operation expenses, and integration of voice and data networks.

Today's IP telephony infrastructure has been enabled by significant investments in research and development. As IP telephony becomes more compatible with the legacy telephony, businesses and individuals find more compelling reasons to migrate to IPbasis telephony. Meanwhile, the high demand for VoIP has fueled business cases for the next generation carriers to build more reliable IP-based voice networks which can also be interconnected to the legacy public switched telephone network (PSTN). In fact, today, the majority of international calling card services are IP-based. With such widespread applications and availability, learning about VoIP, its protocols, and underlying technologies can be considered as valuable academic investment. Consequently, engineering, IT, and technology students who are familiar with these concepts can be ready for the future competitive job market.

Unfortunately, as in many other universities and colleges, at Central Connecticut State University we offer no specific courses on VoIP technology. In fact, in the current networking and IT curriculums, we don't even cover the topic of Voice-over-IP. Consequently, many of our graduates and undergraduate students have very little understanding of VoIP and its underlying technologies.

In this paper we present a simple VoIP laboratory experiment that can be integrated in the classroom. As an introduction to VoIP systems, this experiment can be included in different courses covering advanced networking, Internet technologies, and related topics. Using freely available software, the VoIP experiment presented in this paper can be implemented in any isolated lab environment. Through this experiment, students learn about the basic concepts of IP-based networks, including setting up a voice server and configuring clients, become familiar with network performance parameters (e.g., packet loss and jitter), understand call set-up and tear-down procedure and various signaling protocols involved in such processes, and analyze the impact of traffic volume on voice degradation. In this paper, we also discuss possible extensions to this experiment, including WiFi-based VoIP networks. 


\section{Background}

The Internet is a collection of interconnected networks, all using the Internet Protocol (IP). The Internet protocol is a packet-based protocol in which the traffic is broken into small packets that are sent individually to their destinations. Typically, in the absence of any special solution, the route that each packet takes to reach its destination is determined independently at each network node on a packet-by-packet basis.

Voice over Internet Protocol (VoIP) is simply the transport of voice traffic over the Internet or any IP-based packet-switched network. This is in contrast with the traditional telephone network, carrying voice data over dedicated circuit-switched transmission lines. A major challenge in implementing VoIP is to ensure sufficient bandwidth is available and access to the available bandwidth is controlled and prioritized. Sufficient bandwidth is required to maintain high-quality voice. On the other hand, controlling the bandwidth limits bandwidth-hogging applications and guarantees access for delaysensitive applications.

A major issue with IP, however, is that it does not provide any guarantee of service. Another shortfall of IP is that packets can arrive out of order at their destination. In fact, in extreme cases some packets may be severely delayed or may not arrive at all.

In order to deal with these shortcomings, the transmission control protocol (TCP) has been added to operate along with IP. The primary function of TCP is to ensure error-free delivery of packets to their destination and maintain packets in-sequence. Although, $\mathrm{TCP} / \mathrm{IP}$ protocols have proved to be successful for data transfer, they are not appropriate to deliver voice traffic.

Another choice of protocol that can be used in conjunction with IP is user datagram protocol (UDP). Unlike TCP, UDP does not guarantee in-sequence and error-free packet delivery. Yet, in spite of UDP's unreliable nature, it provides faster packet delivery compared to TCP. For voice communications packet loss of about five percent is generally acceptable. However, voice traffic is very delay sensitive. Consequently, UDP happens to be a proper choice for transporting voice traffic.

UDP was not originally designed for voice traffic. Therefore, in order to overcome some of its shortcomings, without resorting to TCP, a number of protocols, including the realtime transport protocol (RTP) and RTP control protocols (RTPCP) have been developed. For example, in RTP packets include a sequence number to resolve out-of-sequence packet arrivals ${ }^{1}$.

As in traditional phone network, specific signaling protocols are necessary to be invoked before and during a call in order to setup, monitor, and terminate the call. Such protocols are referred to as signaling protocols. In order to ensure interoperatibility between systems from different vendors, the International Union Telecommunications Standardization Section (ITU-T) recommended H.323 protocol to serve as the standard signaling protocol for VoIP. 
The Session Initialization Protocol (SIP) has also been considered as an alternative to H.323 and it is claimed to be less complex, more flexible, and better suited to support advanced features ${ }^{2}$.

There are many advantages associated with implementing VoIP. An obvious advantage of VoIP is the integration of voice and data. This integration can lead to providing a wide variety of features and services. Consider the following potential IP telephony features: calling the customer service by clicking on a button on the company's webpage; being able to have on-screen access to any database while talking on an IP-phone; establishing web/video conferencing with multiple users; providing real phone along with LAN connection to every class room; allowing users to use their phones in the office even when they are away from their desks using WiFi-enabled IP-phones; locating individuals as they move between buildings.

Having a single network to carry voice and data allows users to use their available bandwidth more efficiently. Moreover, VoIP can eliminate the cost of long distance phone calls and compress more calls into available bandwidth than the legacy phone system. The integration of voice and data networks also considerably simplifies the overall network infrastructure, which in turn can result in lower cost of maintenance and operations.

These advantages have attracted many companies and enterprises to consider a systematic migration to VoIP. In 2004, an estimated 4.8 million people used VoIP. The number of VoIP users is expected to grow to over 197 million people by $2010^{10}$. According to Infonetics Research, 36 percent of larger organizations were already using VoIP production and services in $2005^{9}$. Such demands have been the main driving force in attracting many carriers and startup telecommunications companies. Faced with such opportunities, engineering, IT, and technology students, who have been introduced to the basics of IP telephony and its protocols can be very valuable in the future competitive job market.

\section{Classroom Experiment}

Integration of laboratory experiments with class lectures can be an effective approach to teach principle concepts in IP telephony. Such hands-on experiments help students to develop their own simple IP telephony network and teach them about features and capabilities of a VoIP system. There are number of available software that can freely be downloaded and used to setup a VoIP system, such as TrixBox, SIPCAT, SKYPE, and open source VOCAL. A vast number of documents describe the capabilities and setup configuration for each of these software packages ${ }^{11-12}$.

In this experiment we use TrixBox V2.0. We found TrixBox to be less complicated. Moreover, it requires no registration; hence, the software can be installed in an isolated lab environment. We use X-Lite $3.0^{5}$ as the client soft phone. The advantage of X-Lite is that it supports voice and video communications between clients.

There are many different online documents describing how to setup a VoIP network using TrixBox. However, majority of these documents are often very involved and 
complicated to follow in a timely manner, particularly in the classroom environment. Through development of this lab experiment, we focus on a simplified network and attempt to demonstrate the main concepts in VoIP, including signaling protocols, network performance, and call monitoring. Motivated students are encouraged to continue on their own and setup a larger network, examine its advanced features, and find its limitations.

\subsection{Experiment Details}

The hardware and software requirements to perform this experiment are listed in Table 1. Figure 1 shows the server/client network setup required for this laboratory experiment. This experiment is divided into the following three main parts:

a) Installing the hardware and configuring the voice server and clients.

b) Analyzing the voice quality under different network constraints.

c) Monitoring calls as well as examining voice packets and VoIP signaling protocols.

In the following paragraphs we describe details pertaining to each part. The complete lab manual can be retrieved from reference ${ }^{3}$. More details regarding the installation of TrixBox V2.0 can be found in reference ${ }^{4}$.

In the first part of the experiment, each group with two or three students is asked to install TrixBox V2.0 and setup the voice server as instructed in the lab manual. In order to complete this part, the following steps must be executed:

a) Installing TrixBox V2.0 using the provided CD. The installation of the voice server is almost automatic and will take about half an hour.

b) Configuring the voice server and setting up the username and password.

c) Ensuring that the correct Ethernet adaptor has been selected by the software. Otherwise, ifcfg-eth0 or ifcfg-eth0 files must be edited.

d) Changing the sip.conf file to enable the codec support. This is required to enable the video feature in TrixBox V2.0.

e) Installing proper modules in freePBX ${ }^{\mathrm{TM}}$ of TrixBox, including Voicemail, Voice Recording, On Hold Music, etc.

f) Configuring client nodes and assigning a telephone number to each. Client nodes in this experiment are based on Windows XP operating system. We use X-Lite 3.0 soft phone to establish voice/video communications between clients.

At this point, the VoIP network must be ready to operate and students should be able to dial a number and establish a voice/video call between different client nodes.

In the second part of the experiment, students examine the quality of the voice/video calls under various network environments. In this part, students are asked to perform the following changes to the network and observer the voice quality:

a) Change the existing CAT-5 cables with longer cables. 
b) Increase the traffic volume throughout the network; this can be accomplished by continuously invoking ping $-\mathrm{t}$ command.

c) Add more users in order to establish multiple simultaneous connections.

In case of limited available PC, VMware ${ }^{7}$ or Virtual $\mathrm{PC}^{8}$ can be utilized to emulate multiple clients on a single PC.

As an extension to this part, if time permits, we also ask students to modify the network and use a wireless router to interconnect client nodes to the voice server. In this section, students can observe the change in quality of both voice and video as WiFi replaces the LAN. This section of the experiment can be particularly helpful in exposing students to concepts such as interference and channel delays in wireless networks.

Table 1: List of hardware used to setup the VoIP system.

\begin{tabular}{|c|c|}
\hline Hardware / Software & Comments \\
\hline Minimum of three PCs & $\begin{array}{l}\text { One PC must be configured as the server; clients were } \\
\text { configured using Windows XP OS - PC specifications: } \\
\text { - } 250 \mathrm{MHz} \text { Pentium II PC or better } \\
\text { - } 128 \mathrm{MB} \text { RAM - the more the better } \\
\text { - } 4 \text { GB Hard disk space - minimum } \\
\text { - Two or more USB connector } \\
\text { - } 10 / 100 \text { NIC } \\
\text { - CD-ROM Drive } \\
\text { - } 10 / 1004 \text { or } 8 \text { ports Ethernet hub/switch (not } \\
\text { required the router has spare ports) }\end{array}$ \\
\hline $\begin{array}{l}\text { Minimum of two general } \\
\text { purpose USB cameras }\end{array}$ & Used for video conferencing \\
\hline $\begin{array}{l}\text { At least three CAT-5 patch } \\
\text { cables }\end{array}$ & May need more depending on the number of clients used \\
\hline Minimum of two headphones & One will be used for each client \\
\hline One Linksys Router/Switch & $\begin{array}{l}\text { Ensure the compatibility of the router and your } \\
\text { server/client nodes }\end{array}$ \\
\hline TrixBox V2.0 & Voice Server software ${ }^{4}$ \\
\hline X-Lite 3.0 & Client soft phone $^{5}$ \\
\hline VMware or Virtual PC & $\begin{array}{l}\text { VMware allows running Windows on Linux }{ }^{7} \text {; Virtual } \\
\text { PC allows running multiple PCs on a single machine }\end{array}$ \\
\hline Ethereal & $\begin{array}{l}\text { Network protocol analyzer software }{ }^{6} \text { - The later version } \\
\text { of Ethereal is known as Wireshark. Wireshark is } \\
\text { considered to be one of the best open-source network } \\
\text { analyzers available. }\end{array}$ \\
\hline
\end{tabular}

In the third part of the experiment, we ask students to install a network protocol analyzer, namely Ethereal, and monitor voice and video packets traveling between clients and the server as calls are made. Ethereal allows students to capture traces. Using the function Analyze $\rightarrow$ VoIP all the transmitted messages during the call setup can be collected, read, and examined. This part of the laboratory experiment is designed to demonstrate how to 
troubleshoot the network. For example, students can examine the network and see why the server is not properly responding to client requests and dropping their requests or whether the server is properly acting as a Proxy for clients.

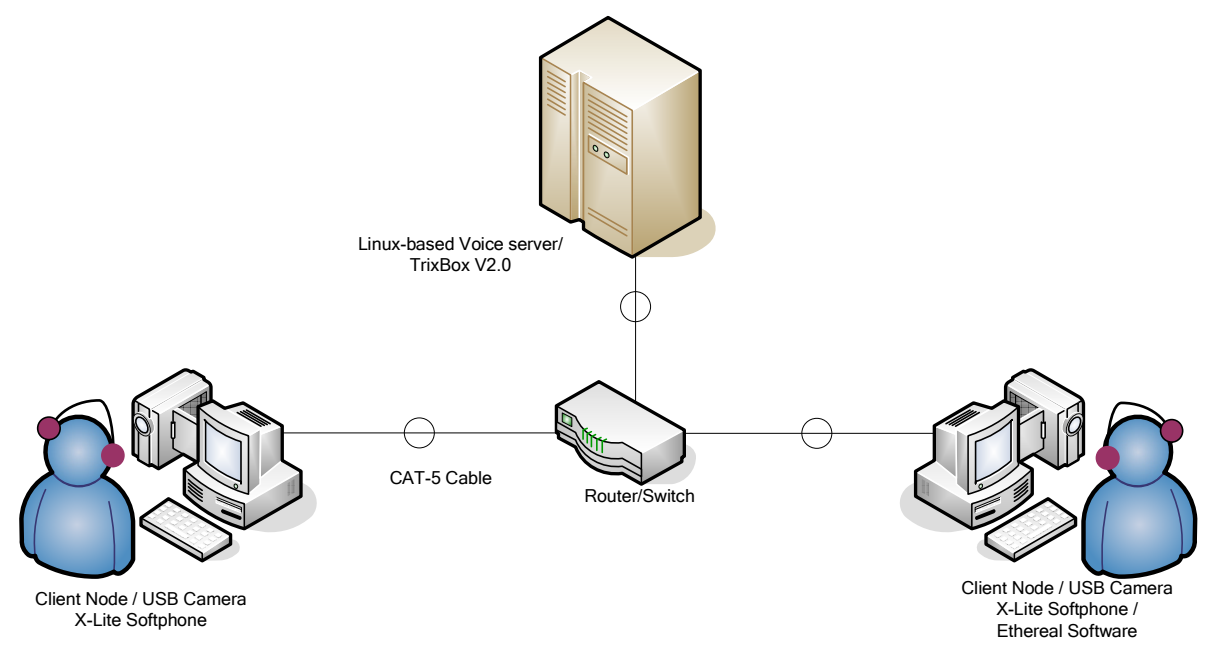

Figure 1: Network configuration of the VoIP system.

\subsection{Extended Laboratory Experiments}

Interested students can enhance their skills by examining more advanced functionalities provided by TrixBox V2.0. For example, students can implement various modules offered by the freePBX TM, including paging and queues. Furthermore, students can establish conference calls between multiple users and examine the voice quality and limitations of the network. The TrixBox can also be configured for H.323 signaling protocols. Using Ethereal, students can spend a good deal of time examining the difference between H.323 and SIP signaling protocols and learn about their differences and similarities. Another interesting extension to this laboratory experiment can be setting up VoIP gateway such that multiple VoIP networks are interconnected together.

\section{Experiment Outcomes}

Our main motivation in developing this experiment is to provide an introductory section on VoIP technology that can be added to networking and Internet technology courses across different programs, including Computer Science (CS), Electronic Engineering (EE), Computer Engineering Technology (CET), and IT. This section is developed primarily for $4^{\text {th }}$ and $5^{\text {th }}$ year undergraduate and $1^{\text {st }}$ year graduate students enrolled in CS, CET, and IT programs.

\subsection{Assessment Results}

The VoIP experiment was integrated in both sections of CET479. This course focuses on various client/server Internet-based technologies. Students in this course often spend one- 
third of their time in the laboratory in order to complete their lab assignments. The VoIP described above was assigned to both sections following the classroom lectures.

At the end of the semester, the students were asked to complete a mandatory short survey for the course. The survey was primarily based on quantitative questions with the last question asking for student comments and suggestions. The combined demographics of the two sections were as follow: $20 \% \mathrm{CS}, 50 \%$ IT, 22\% CET, and $8 \%$ CIT (graduates). The responses to some of the quantitative questions concerning the VoIP laboratory experience are shown in Table 2 .

Table 2. Student responses to the course survey.

\begin{tabular}{|c|c|c|c|c|c|c|}
\hline \multicolumn{7}{|c|}{$\square$ The VoIP lab experiment was } \\
\hline \multirow[b]{2}{*}{ Responses $\rightarrow$} & \multicolumn{2}{|c|}{ Too simple } & \multicolumn{2}{|c|}{ About right } & \multicolumn{2}{|c|}{ Too Complex } \\
\hline & \multicolumn{2}{|c|}{$0 \%$} & \multicolumn{2}{|c|}{$72 \%$} & \multicolumn{2}{|c|}{$28 \%$} \\
\hline \multicolumn{7}{|c|}{$\begin{array}{l}\square \text { The VoIP experiment helped me to enhance my understanding of IP telephony } \\
\text { concepts }\end{array}$} \\
\hline \multirow[b]{2}{*}{ Responses $\rightarrow$} & \multicolumn{2}{|c|}{ Strongly agree } & Agree & Disagree & \multicolumn{2}{|c|}{ Strongly disagree } \\
\hline & \multicolumn{2}{|c|}{$35 \%$} & $55 \%$ & $10 \%$ & \multicolumn{2}{|c|}{$0 \%$} \\
\hline \multicolumn{7}{|c|}{$\square$ The time dedicated to complete the VoIP lab experiment was } \\
\hline \multirow[b]{2}{*}{ Responses $\rightarrow$} & \multicolumn{2}{|c|}{ Too Short } & \multicolumn{2}{|c|}{ About right } & \multicolumn{2}{|c|}{ Too long } \\
\hline & \multicolumn{2}{|c|}{$20 \%$} & \multicolumn{2}{|c|}{$50 \%$} & \multicolumn{2}{|c|}{$30 \%$} \\
\hline \multicolumn{7}{|c|}{$\square$ The VoIP experiment should be continued in future semesters } \\
\hline \multirow[b]{2}{*}{ Responses $\rightarrow$} & \multicolumn{2}{|c|}{ Strongly agree } & Agree & Disagree & \multicolumn{2}{|c|}{ Strongly disagree } \\
\hline & \multicolumn{2}{|c|}{$30 \%$} & $61 \%$ & $9 \%$ & \multicolumn{2}{|c|}{$0 \%$} \\
\hline \multicolumn{7}{|c|}{$\begin{array}{l}\text { Students must be given more time to complete advanced features in the VoIP lab } \\
\text { experiment }\end{array}$} \\
\hline \multirow[b]{2}{*}{ Responses $\rightarrow$} & \multicolumn{2}{|c|}{ Strongly agree } & Agree & Disagree & \multicolumn{2}{|c|}{ Strongly disagree } \\
\hline & \multicolumn{2}{|c|}{$10 \%$} & $62 \%$ & $19 \%$ & \multicolumn{2}{|c|}{$9 \%$} \\
\hline \multicolumn{7}{|c|}{$\square$ Which of the topics covered in this course would you likely to explore further? } \\
\hline \multirow[b]{2}{*}{ Responses $\rightarrow$} & VoIP & Cluster & $\begin{array}{c}\text { Web } \\
\text { Security }\end{array}$ & $\begin{array}{l}\text { Web } \\
\text { Monitoring }\end{array}$ & Others & None \\
\hline & $27 \%$ & $13 \%$ & $10 \%$ & $20 \%$ & $20 \%$ & $10 \%$ \\
\hline
\end{tabular}

In their comments, many students indicated that they would like to see more real-life examples of VoIP applications. Some students believed that instructions listed in the lab handouts were not very clear. In fact, throughout the experiment, the students were very active in providing corrections and their feedbacks greatly assisted us in updating lab handouts.

In regards to lab equipments, some students expressed concern that their machines and peripherals, including sound cards, web cams, and microphones, were not fully functional and they lost considerable lab time. In general, most students expressed satisfactions with their lab partners and found her/him helpful in completing the experiment.

Based on our survey, almost 90 percent the students who implemented their own VoIP network found the laboratory exercise interesting and educational. Students with more 
knowledge about TCP/IP operation spend more time tracing packets and learning about VoIP signaling protocols. They were also very eager to learn more about setting up a voicemail and performing call monitoring. For less experienced students, this laboratory experiment was ideal to gain first-hand experience in setting up and configuring a server and observing how a server/client network operates.

\section{Conclusion}

In this paper we presented a practical demonstration of VoIP that can be implemented in an isolated classroom environment. We utilized a freely available Linux-based software that allows students to setup an IP-based telephony system. The laboratory experiment can be incorporated in any networking or Internet technology class covering related areas.

Student responses to the quantitative and free-form questions concerning the implementation of the VoIP system indicated that majority of students found the experiment interesting and helpful in enhancing their learning. The complexity of the experiment appeared to be satisfactory to most students and, overall, their feedback was positive. Based on students' feedbacks, we learned that a number of measurements, including improvement of instructions in the lab manual and lab equipment, can further improve their learning experience.

\section{Acknowledgment}

The author would like to thank Mr. Davy Jagan for his valuable contributions and efforts in setting up and testing this experiment at the Advanced Internet Technology in the Interests of the Society (AITIS) laboratory at Central Connecticut State University. 


\section{References}

1. D. Collins, Carrier Grade Voice Over IP. Second ed. McGraw-Hill publishing, September 2000. ISBN: 0071363262.

2. B. Douskalis, Putting VoIP to Work: Softswitch Network Design and Testing. 1st edition Prentice Hall PTR Publishing, September 2001. ISBN: 0130409596.

3. Refer to www.ccsu.edu/technology/farahmand/ for complete step-by-step instructions to setup a VoIP network using TrixBox V2.0 and X-Lite 3.0.

4. TrixBox Configuration Guide, 2006. Retrieved from www.trixbox.org on December 12, 2006.

5. X-Lite can be downloaded from www.xten.com .

6. A. D. Orebaugh, G. Morris, and E. G. Ramirez, Ethereal Packet Sniffing, Syngress Publishing; 2004. ISBN: 1932266828.

7. VMware, Retrieved from http://en.wikipedia.org/wiki/VMware on December, 2006.

8. Virtual PC. Retrieved from http://www.microsoft.com/windows/virtualpc/default.mspx on December, 2006.

9. L. Erlanger, Building VoIP into the Enterprise, InfoWorld. Retrieved from http://www.infoworld.com/article/06/06/22/79155_26FEvoipstrat_1.html on December 12, 2006.

10. M. Matt, Experts Say Applications Will Drive VoIP Growth. Nov. 2005, eWeek, Retrieved from www.eweek.com/article2/0,1895,1861513,00.asp on December 12, 2006.

11. D. G. Kelly, C. Jennings, and L. Dang, Practical VoIP Using VOCAL. ISBN: 0596000782.

12. W. A. Fryer, Tools for the Teks: Integrating Technology in the Classroom. Retrieved from www.wtvi.com/TEKS/05_06_articles/classroom-audiopodcasting.html, on December 12, 2006.

\section{Bibliography}

FARID FARAHMAND is an Assistant Professor in the School of Engineering and Technology at Central Connecticut State University where he teaches Advanced Networking and Digital Systems. Farid's research interests are optical networks and optical burst switching, including their architecture and performance. He is also the director of Advanced Internet Technology in the Interests of Society Laboratory. 